\title{
Modern challenges in acute coronary syndrome
}

\author{
Despite a growing evidence base, gaps in knowledge and practice leave room \\ for improvement in the treatment of acute coronary syndrome
}

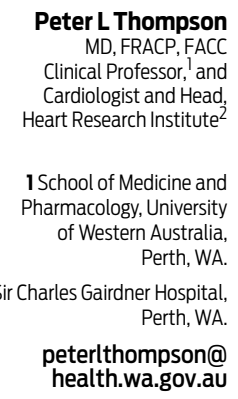

doi: 10.5694/mja14.01468 few decades ago, there was still controversy about the importance of interruption of blood flow versus myocardial tissue oxygen demand in causing myocardial infarction..$^{1,2}$ It is now universally accepted that coronary thrombosis at the site of an unstable atherosclerotic plaque is the usual cause of coronary occlu$\operatorname{sion}^{3}$ and the cluster of conditions of unstable angina, non-ST-elevation myocardial infarction (NSTEMI) and ST-elevation myocardial infarction (STEMI) comprise the clinical complex now called acute coronary syndrome (ACS).

An important observation from the investigations at the start of the "reperfusion era" was the recognition that STEMI and NSTEMI, while both due to coronary thrombosis, had quite different presentations and natural histories. ${ }^{4}$ Important differences between the pathophysiology of STEMI and NSTEMI determine the focus of treatment. In STEMI, the complete occlusion of the coronary vessel initiates a cascade of myocardial necrosis, which can be prevented by early reperfusion with percutaneous coronary intervention or fibrinolytic therapy. ${ }^{5}$ In NSTEMI, the less complete occlusion of the coronary vessel means there is less immediate urgency to salvage myocardium, and the initial focus is on antithrombotic therapy to limit the size and instability of the thrombosis in the coronary artery. In this situation, the size, shape and location of the coronary thrombosis are highly variable. The patient's clinical course can be unpredictable, and progression to STEMI is a pervading concern. In patients with NSTEMI who are at high risk, an early invasive approach has been shown to be superior to a conservative approach, ${ }^{6}$ but the optimal timing of this remains controversial. ${ }^{7}$ These major advances in understanding this symptom complex have driven quantum shifts in management approaches and greatly improved outcomes for patients who have suffered a heart attack. However, it remains a condition which can be unpredictable and, despite the best of modern treatments, can still be lethal. As ACS is a symptom of underlying coronary heart disease, longterm management is often more important than the acute phase. This supplement focuses on the many challenges in managing ACS.

The first two articles in this supplement deal with managing the acute stage of ACS. The many valuable guidelines on this topic, ${ }^{8-12}$ not reiterated in detail in the supplement, all concur on the basics of modern therapy. The use of potent antithrombotic agents is central to tackling the coronary thrombosis, albeit with an increased risk of bleeding. While controversies continue over the ideal duration of antiplatelet therapy, the evidence to support routine early and post-hospital use of potent antiplatelet agents is overwhelming. Statin therapy is also central to the management of the acute episode and for long-term management, irrespective of the low-density lipoprotein cholesterol level at the time of the episode. The role of $\beta$-adrenergic blockers and inhibitors of the renin-angiotensin-aldosterone system remain important, but perhaps better targeted to patients at higher risk. The guidelines, while sometimes exhaustingly complete, do not cover all aspects of management.

In the first article in the supplement, Brieger focuses on the identification of patients with ACS who are at high risk (page S88). He argues that routine risk stratification as soon as possible after presentation will determine the clinical pathway, and that this practice should be embedded in the hospital system - it is too important to leave to ad-hoc and potentially unreliable clinical judgement. This is a challenging change in approach for the hospital system, but bound to be fruitful in reducing decision time when early revascularisation is needed, and avoiding unnecessary intervention when it is not.

Next, McQuillan and Thompson review the limited evidence to guide management in four important subgroups: female, older, diabetic and Indigenous patients (page S91). These subgroups have been underrepresented in clinical trials, in contrast with the evidence base that guides the care of most other patients with ACS, which is rich and detailed. There is also evidence that these subgroups are at particular risk, and clinical decisions must often be based on extrapolation from the results of clinical trials without absolute certainty that the evidence is applicable.

The other articles in the supplement deal with the challenges in caring for post-ACS patients at the time of discharge from hospital and handover to the general practitioner. This transition can lead to confusion for the patient and frustration for the GP in dealing with patients returning to their practice with major changes in their management incompletely documented and uncertainty about how best to access the services available to their patients.

Redfern and Briffa use data from three registries to describe common shortfalls in the transition from hospital to primary care (page S97). The challenges in improving access to effective secondary prevention are concisely summarised, with positive guidance on how to improve secondary prevention in primary care, raising awareness of the need for lifelong secondary prevention, better integration and use of existing services, consideration of the use of registry data in data monitoring and quality assurance, and the potential in embracing new technologies such as automated texting reminders to patients, already outlined in a summit on this topic last year. ${ }^{13}$

Thompson and colleagues summarise the extensive evidence base for ideal post-hospital therapy (page S100), focusing on the $50 \%$ of patients who do not receive coronary 
intervention or revascularisation at the time of their acute episode. ${ }^{14}$ The extensive collaboration on clinical trials and registries that has gone into developing the rich evidence base is a source of pride in modern cardiology, but many gaps in evidence remain.

Thakkar and Chow reassert the truism that drugs do not work in patients who do not take them (page S106); there is evidence that non-adherence among post-ACS patients is common and associated with adverse outcomes. ${ }^{15}$ Their review summarises strategies to improve adherence to prescribed medications, and touches on the future possibility of a polypill to include a combination of evidence-based therapies to improve adherence.

Finally, Vickery and Thompson take the GP's perspective in managing the post-ACS patient and describe eight common challenges that GPs face in this setting (page S110). The need for courteous, detailed communication between the hospital and primary care is highlighted.

The common theme of each article in this supplement is that progress has been impressive, but much has to be done to continue the improvements in understanding and in translating the knowledge we already have into further improvements in outcomes. The disturbing evidence from recent Australian nationwide surveys that the application of proven evidence-based therapies remains less than optimal ${ }^{16}$ is a concern and presents a major challenge in the modern management of ACS.

Competing interests: I have received research funding from AstraZeneca, Boehringer Ingelheim, Pfizer, Bristol-Myers Squibb and Amgen and advisory board fees from AstraZeneca and Pfizer.

Provenance: Commissioned; not externally peer reviewed.

1 Spain DM, Bradess VA. The relationship of coronary thrombosis to coronary atherosclerosis and ischemic heart disease (a necropsy study covering 25 years). Am J Med Sci 1960; 240: 701-709.

2 Roberts WC, Buja LM. The frequency and significance of coronary arterial thrombi and other observations in fatal myocardial infarction: a study of 107 necropsy patients. Am J Med 1972; 52: 425-443.

3 Davies MJ. Stability and instability: two faces of coronary atherosclerosis. The Paul Dudley White Lecture 1995. Circulation 1996; 94: 2013-2020.

4 Fibrinolytic Therapy Trialists' (FTT) Collaborative Group. Indications for fibrinolytic therapy in suspected acute myocardial infarction: collaborative overview of early mortality and major morbidity results from all randomised trials of more than 1000 patients. Lancet 1994; 343: 311-322.

5 Van de Werf F. The history of coronary reperfusion. Eur Heart J 2014; 35: 2510-2515.

6 Fox KA, Clayton TC, Damman P, et al; FIR Collaboration. Long-term outcome of a routine versus selective invasive strategy in patients with non-ST-segment elevation acute coronary syndrome: a meta-analysis of individual patient data. J Am Coll Cardiol 2010; 55: 2435-2445.

7 Navarese EP, Gurbel PA, Andreotti F, et al. Optimal timing of coronary invasive strategy in non-ST-segment elevation acute coronary syndromes: a systematic review and meta-analysis. Ann Intern Med 2013; 158: 261-270.

8 Hamm CW, Bassand JP, Agewall S, et al. ESC Guidelines for the management of acute coronary syndromes in patients presenting without persistent ST-segment elevation: the task force for the management of acute coronary syndromes (ACS) in patients presenting without persistent ST-segment elevation of the European Society of Cardiology (ESC). Eur Heart J 2011; 32: 2999-3054.

9 Anderson JL, Adams CD, Antman EM, et al. 2012 ACCF/AHA focused update incorporated into the ACCF/AHA 2007 guidelines for the management of patients with unstable angina/non-ST-elevation myocardial infarction: a report of the American College of Cardiology Foundation/American Heart Association Task Force on Practice Guidelines. Circulation 2013; 127: e663-e828.

10 Chew DP, Aroney CN, Aylward PE, et al. 2011 Addendum to the National Heart Foundation of Australia/Cardiac Society of Australia and New Zealand Guidelines for the management of acute coronary syndromes (ACS) 2006. Heart Lung Circ 2011; 20: 487-502.

11 Task Force on the management of ST-segment elevation acute myocardial infarction of the European Society of Cardiology (ESC), Steg PG, James SK, et al. ESC Guidelines for the management of acute myocardial infarction in patients presenting with ST-segment elevation. Eur Heart J 2012; 33: 2569-2619.

12 O'Gara PT, Kushner FG, Ascheim DD, et al. 2013 ACCF/AHA guideline for the management of ST-elevation myocardial infarction: a report of the American College of Cardiology Foundation/American Heart Association Task Force on Practice Guidelines. Circulation 2013; 127: e362-e425.

13 Redfern J, Chow CK; Executive Committee and participants in the National Secondary Prevention of Coronary Disease Summit, 2011. Secondary prevention of coronary heart disease in Australia: a blueprint for reform. Med J Aust 2013; 198: 70-71.

14 Chew DP, French J, Briffa TG, et al. Acute coronary syndrome care across Australia and New Zealand: the SNAPSHOT ACS study. Med J Aust 2013; 199: 185-191.

15 Chew DP, Anderson FA, Avezum A, et al. Six-month survival benefits associated with clinical guideline recommendations in acute coronary syndromes. Heart 2010; 96: 1201-1206.

16 Redfern J, Hyun K, Chew DP, et al. Prescription of secondary prevention medications, lifestyle advice, and referral to rehabilitation among acute coronary syndrome inpatients: results from a large prospective audit in Australia and New Zealand. Heart 2014; 100: 1281-1288. 